\title{
SISTEMAS DE ACONDICIONAMIENTO DE AIRE Y VENTILACIÓN COMO FOCO INFECCIOSO DE SARS CoV-2 EN HOSPITALES, CLÍNICAS E INSTITUCIONES DE SALUD
}

\author{
Waldyr Fong Silva ${ }^{1 *}$, Carlos Alberto Severiche Sierra ${ }^{2}$, Wendy Marcela Fong Amarís ${ }^{3}$ \\ ${ }^{1,2,}$ Universidad de Cartagena. Cartagena de Indias, Colombia \\ ${ }^{3}$ Pontificia Universidad Javeriana. Bogotá, Colombia \\ *Autor de correspondencia: wfongs@ unicartagena.edu.co
}

Recibido Abril 2020; Aceptado Junio 2020

\begin{abstract}
Resúmen - En el presente trabajo se hace un análisis de la forma como los sistemas de acondicionamiento de aire, calefacción y ventilación se convierten en posibles focos infecciosos de transmisión del Sars Cov-2 debido a los ventiladores internos con los que cuentan y que generan la corriente de aire forzada que refresca, calienta o ventila el recinto o habitación. También se prsentan 4 elementos sobre los cuales se deben hacer las auditorías en hospitales y centros de salud para que no se conviertan en fuentes infecciosas, como lo son: a) diseño de condiciones ambientales según los criterios de Ashrae 170-2013, Ashrae 55; b) ventilación adecuada del recinto según los criterios de Ashrae 62; c) renovación y suministro de aire fresco exterior hacia los recintos según criterios de la norma Ashrae 62 y DIN 1946; d) requerimientos mínimos de diseño de los sistemas de filtración de este tipo de dispositivos según criterios de la norma EN 1822:2009 e ISO 29463. Se concluye que los sistemas de calefacción, ventilación y aire acondicionado, ocasionan la movilidad de núcleos de gotas y gotitas respiratorias en el ambiente convirtiéndose en fuente primaria de transmisión del Sars Cov-2 y que para evitarlo, se hace necesario utilizar sistemas de tratamiento de aire que posean filtros de alta eficiencia HEPA y en lo posible utilicen cámaras germicidas con luz ultravioleta.
\end{abstract}

Palabras Clave: Sars Cov-2, aire acondicionado, ventilación, renovación de aire, filtración, ultravioleta, normas.

\section{AIR CONDITIONING AND VENTILATION SYSTEMS AS AN INFECTIOUS FOCUS OF SARS CoV-2 IN HOSPITALS, CLINICS AND INSTITUTIONS OF HEALTH}

\begin{abstract}
In the present work, an analysis is made of the way in which the air conditioning, heating and ventilation systems become possible infectious sources of transmission of Sars Cov-2 due to the internal fans that they have and that generate the current of forced air that cools, heats or ventilates the enclosure or room. There are also 4 elements on which audits should be made in hospitals and health centers so that they do not become infectious sources, such as: a) design of environmental conditions according to the criteria of Ashrae 170-2013, Ashrae 55; b) adequate ventilation of the enclosure according to the criteria of Ashrae 62; c) renewal and supply of fresh outside air to the premises according to the criteria of the Ashrae 62 and DIN 1946 standards; d) minimum design requirements of the filtration systems of this type of devices according to the criteria of the EN 1822: 2009 and ISO 29463 standards. It is concluded that heating, ventilation and air conditioning systems cause the mobility of droplet nuclei and respiratory droplets in the environment, becoming a primary source of transmission of Sars Cov-2 and that to avoid this, it is necessary to use treatment systems for air with high-efficiency HEPA filters and where possible use germicidal chambers with ultraviolet light.
\end{abstract}

Keywords - Sars Cov-2, air conditioning, ventilation, air renewal, filtration, ultraviolet, standards. 


\section{Introducción}

Es común encontrar en clínicas, hospitales, consultorios odontológicos, EPS (entidades prestadoras de salud) e instituciones de salud de ciudades a lo largo del planeta, sistemas de aire acondicionado Tipo Split o piso techo en sala de urgencias, unidad de cuidados intensivos (UCI), consultorios y habitaciones. Así mismo, también podemos encontrar sistemas de aire acondicionado central con sistemas de ductería, suministro y retorno de aire. En todos éstos sistemas, incluyendo los de calefacción, el equipo recircula de forma continua el aire de la habitación o recinto hasta que se acondiciona a una temperatura de confort media comprendida entre los 18 y $24{ }^{\circ} \mathrm{C}$ y humedad relativa entre 50 y $60 \%$. En otros, el diseño es deficiente y no alcanza ni la humedad relativa ni la temperatura media de confort estipulada por la normativa internacional. En ambos casos, independiente de si se alcanzan o no las condiciones de confort, los equipos cuentan con ventiladores que inyectan y extraen continuamente aire de la habitación generando corrientes con partículas en suspensión y posibles portadoras del patógeno. Esto implica que las corrientes de aire producidas por estos equipos puedan ser un foco infeccioso y de contagio masivo de SARS-CoV-2 y que a la fecha no ha sido evaluado. Estos diferentes mecanismos de contagio sin capacidad de los gobiernos para contenerlo constituye en la actualidad una amenaza a la salud pública mundial (Cortés, 2020) en donde el desafío para educar y controlar la epidemia se convierte en un reto de enormes proporciones tanto para países desarrollados como no desarrollados (Moreno, 2020). Toda esta situación de contagio afecta a todo tipo de población desde la más joven hasta la más anciana, es decir, no hay límite de edad para el contagio por Sars-Cov-2 (Lu et al. (2020) sin conocer aún, el origen de este virus y si contagia además, a otro tipo de animales en la naturaleza (Andersen et al. (2020; Ludert \& Franco, 2020).

Por lo anterior, la primera intervención que se debe hacer en este tipo de instituciones de salud es revisar en cada zona (UCI, urgencias, quirófanos, cuidados intermedios entre otras) las condiciones de diseño y confort térmico establecidas internacionalmente por ASHRAE 55 (De Dear, 2002; Olesen \& Brager, 2004) y que corresponden a $24{ }^{\circ} \mathrm{C} \pm 1{ }^{\circ} \mathrm{C}, 50 \% \pm 5 \%$ HR y velocidad del aire de $0,5 \mathrm{~m} / \mathrm{s}$ (100fpm). Así mismo, debe revisarse el cumplimiento de los lineamientos establecidos en el Estándar 170-2013 de ASHRAE/ASHE (ASHRAE 170, 2013) para evaluar si se cumplen o no los requerimientos mínimos de diseño relacionados con la filtración (ASHRAE 52, 2017; Zhou \& Shen, 2007), ventilación y suministro de aire fresco exterior (ASHRAE 62; Persily, 2015; Moschandreas \& Vuilleumier 1999). Todo estos elementos, implican riesgos laborales en los hospitales, clínicas e instituciones de salud ya que el funcionario de la salud se encuentra expuesto continuamente a estos flujos de aire en cada una de las secciones ya sea UCI, cuidados intensivos, consultorios, sala de partos, cirugía entre otras (Ahumada et al., 2016; Herrán et al., 2016; Marrugo, 2017; Morales, 2018; Palacio et al., 2018; Ahumada et al., 2019; Quintana et al., 2019)

Adicionalmente al diseño de las condiciones de temperatura y humedad es importante también prestar atención al diseño de los sistemas de ventilación, renovación de aire y filtración. En este sentido, el aire de ventilación lo conforman 2 elementos que son: aire exterior más cualquier aire recirculado que ha recibido

IPSA SCIENTIA - Vol. 5 № $1-2020$ 
tratamiento para mantener la calidad de aire interior deseado. El volumen mínimo requerido de este aire en cfm (pies $3 / \mathrm{min}$ ) según ASHRAE 62 para algunas zonas de hospitales como pasillos, comedores, salas de cirugía y parto, anfiteatros, fisioterapia, laboratorios de trabajo pesado entre otras zonas son: 20, 35, 20, 10, 15 y 15 respectivamente. Esto indica que cada zona debe ser evaluada según éstos y otros criterios adicionales que tiene la norma y que deberán cumplirse para garantizar el volumen de aire adecuado que mantenga las concentraciones de contaminantes (partículas, dióxido de carbono) en los valores permisibles. De la misma manera, el volumen de cada zona del hospital, debe renovarse continuamente de acuerdo con los criterios de normas internacionales como la ASHRAE o la DIN 1946 en donde se sugieren entre 5 y 6 renovaciones de aire por hora $(\mathrm{N})$. Es decir, para una habitación de dimensiones $3 \times 3 \times 3=27 \mathrm{~m}^{3}$, se requerirá un caudal $Q$ mínimo de aire exterior de: $\mathrm{Q}=\mathrm{N}(27)=6 \times 27=162 \mathrm{~m}^{3} / \mathrm{h}=95.2 \mathrm{cfm}$ y a partir de este valor se procede con el diseño de ventilación o acondicionamiento de aire adecuado para la habitación. Precisamente, este es el diseño que toca revisar en hospitales, clínicas e instituciones de salud para evaluar si el volumen de aire se renueva en su totalidad o si, por el contrario, aquel volumen que no se logre renovar se convierte en posible foco infeccioso y transmisor del virus SARS-CoV-2.

Para los sistemas de filtración, la Sociedad Americana de Ingenieros en Calefacción, Refrigeración y Aire Acondicionado (ASHRAE) diseñó la métrica MERV, que significa mínimo rendimiento de la unidad de filtración y que varía de 1 a 20 en donde a medida que aumenta el número, los filtros se hacen más eficientes. Los filtros de aire HVAC con una clasificación MERV entre 1 y 4 capturan material particulado superior a las 10 micras entre los que se encuentran polen, ácaros, fibras textiles, es decir, ofrecen mínima filtración. Los filtros comprendidos entre 13 y 16 pueden retener partículas con tamaños comprendidos entre 0.3 y 1 micra, es decir, pueden retener partículas como humo de tabaco, bacterias y partículas de estornudo. Sin embargo, su eficiencia es parecida a la de los tapabocas N95 cuya capacidad de filtración es de al menos el $95 \%$ de partículas de tamaño 0.3 micras en un ambiente saturado.

Es importante recordar que según los investigadores Palacios et. al (2020), Van Doremalen et al. (2020), Wang et al. (2020), Kissler et al. (2020), Dong et al. (2020), el tamaño promedio del virus SARS-CoV-2 oscila entre 0.08 y 0.12 micras, es decir, los filtros anteriormente anotados no podrían detener el número de contagios. Para evitarlos, se requieren filtros tipo HEPA cuya clasificación la encontramos en las normas EN 1822:2009 e ISO 29463 y son los indicados para partículas suspendidas con tamaños inferiores a una micra $(<1 \mu \mathrm{m})$ y en donde podría ir adherido el virus ya que su tamaño se asocia con esta categoría $(0.16$ $<1 \mu \mathrm{m}$ ). Entonces la clase de filtro HEPA consistente sería el E10, E11 y E12 (gérmenes, bacterias, virus, humo de tabaco,humo de óxido metálico), H13 (humos de aceite, hollín en estado naciente, partículas radiactivas suspendidas) H14 (ISO 45Hd), U15 (ISO 55U) y U16 (ISO 65U) (aerosoles de todo tipo incluye los aerosoles virales). Entre éstos, los H14, U15 y U16 son los más eficientes con eficiencias superiores a $\geq 99.995 \%, \geq 99.9995 \%$ y $\geq 99.99995 \%$ respectivamente. Por esta razón, se debe hacer una auditoria en hospitales, clínicas e instituciones de salud de la ciudad y el país, que evalúe la calidad del aire interior y mida el tamaño de partículas presentes en cada una de las zonas que se manejan en estas

IPSA SCIENTIA - Vol. 5 № 1 - 2020 
instituciones con el objeto de conocer si cuentan con un sistema de filtrado óptimo para partículas en suspensión y aerosoles virales que permita detener la proliferación del SARS-CoV-2.

De acuerdo con el informe de la OMS titulado "Modos de transmisión del virus que causa la COVID-19" (OMS, 2020), las infecciones respiratorias se pueden transmitir mediante núcleos de gotas (tamaños inferiores a $\mathbf{5} \boldsymbol{\mu m}$ ), gotitas respiratorias (tamaños superiores a $\mathbf{5}$ - 10 micras ) y mediante fómites (objetos contaminados con el patógeno). Para el primer caso, éstas pueden permanecer en el aire durante largos períodos de tiempo y ser transmitidas a distancias superiores a un metro. Por esta razón, es importante prestar especial atención al flujo de aire acondicionado, de calefacción o ventilación que se generan en este tipo de equipos y que pueden estar ocasionando la transmisión masiva del virus. Esto ha podido comprobarse en algunos estudios como el hecho por Santarpia (2020), Barrios (2020), Pan et al. (2020), Kissler et al. (2020) en 13 pacientes aislados y diagnosticados positivos para SARS-CoV-2, verificó la presencia del virus en artículos personales de los pacientes en un $76.5 \%$ de las muestras, en el aire de la habitación en un $63.2 \%$ y en muestras de aire de pasillos en un $66.7 \%$.

Para Tellier (2019), Bourouiba (2020), Serra (2020), Franco (2003), García (2020), Zou et al. (2020), Paules et al. (2020), Huang et al. (2020), Trilla (2020), Sia et al. (2020), Tong et al. (2020), Yeo et al. (2020) el virus se halló también en muestras de teléfonos celulares $(77.8 \%)$, en controles remotos para televisores (55.6\%), en los inodoros de la habitación (81.0\%), superficies de las habitaciones muestreadas $(75.0 \%)$, mesas de noche y barandas $(70.8 \%)$, repisas $(72.7 \%)$, piso debajo de las camas de los pacientes, rejillas de ventilación e inclusive otros pasillos alejados, fueron positivos en un $58.3 \%$. Todos los casos fueron monitoreados en la unidad de biocontención de Nebraska (NBU) para los pacientes que requerían hospitalización y en la NQU (Unidad de cuarentena nacional) para los que presentaban síntomas leves o eran asintomáticos positivos y donde adicionalmente todas las habitaciones NBU y NQU se encontraban diseñadas bajo los criterios de presión negativa, es decir, al ingresar o salir personal de la habitación, el aire del exterior entra y en ninguna circunstancia el aire interior sale de ella (Beam, 2014).

Aunque en los estudios hechos por Johnson et. al (2011), Lee et. al (2019) y Van Doremalen et. al (2020) no utilizaron ninguna técnica para determinar el tamaño de las gotas y partículas con SARS-CoV-2, los datos sugieren que los aerosoles virales fueron producidos por individuos positivos para SARS-CoV-2 en ausencia de tos, las cuales fueron transportadas hasta los pasillos tanto por el personal de muestreo como por aerosoles virales suspendidos o re-suspendidos en las corrientes de aire de los sistemas de ventilación o de acondicionamiento de aire. Este aerosol expirado por humanos se produce en diferentes tipos de actividades como respirar, hablar, toser y aumenta con el nivel de contagio. Además, este aerosol infeccioso puede persistir durante varias horas y tiene un diámetro inferior a las $10 \mu \mathrm{m}$ pudiendo en ese lapso de tiempo ser transportado por las corrientes de aire de la ventilación natural o forzada generada por los equipos de acondicionamiento de aire.

Por otro lado, de acuerdo con los estudios hechos por Santarpia (2020) y Tellier et. al (2019), en la unidad NBU, donde la movilidad de los pacientes era bastante restringida, la distribución del virus en las

IPSA SCIENTIA - Vol. 5 № 1 - 2020 
diferentes muestras tomadas de objetos y sitios de la habitación, sugiere una fuerte influencia del flujo de aire. El modelado de flujo de aire adelantado por estos investigadores logró comprobar que el "tiro del sistema de aire" es el causante de la deposición de las partículas contaminadas a lo largo de toda la habitación e inclusive fuera de ella. La preocupación por conservar y mejorar la Calidad del Aire Interior se ha convertido actualmente en un reto ya que a través de él se transmiten esporas, hongos, virus, bacterias y otros patógenos, lo que hace que reflexionemos al respecto, debido a la emergencia sanitaria por la que atravesamos.

En China Li Y et. al (2020) simularon con gases en un restaurante la circulación de aire en un salón en donde se encontraba una persona positiva con el SARS-CoV-2 y que transmitió la infección a varias de las personas que se encontraban en el mismo sitio y cercanas a él. Con esta técnica lograron comprobar que la enfermedad se transmitió por medio de aerosoles virales exhalados por la persona portadora y que se suspendieron en el aire. Aquellas personas que se encontraban en salones aledaños por los que no se circuló el aire, no resultaron infectadas.

Todo lo anterior nos permite suponer que los sistemas de calefacción, ventilación y aire acondicionado, los cuales poseen ventiladores en sus diferentes sistemas para la circulación de aire, podrían estar ocasionando la movilidad de núcleos de gotas y gotitas respiratorias en el ambiente al cual acondicionan. De la misma manera, éstas corrientes de aire forzado pueden también poner en suspensión aquellas partículas contaminadas con el virus que se encuentren depositadas en fómites. Esto implica que en hospitales, clínicas e instituciones de salud sea necesario reforzar estos equipos (sistemas de calefacción, ventilación y aire acondicionado) con sistemas de limpieza y desinfección mediante soluciones de tratamiento de aire que posean purificadores de aire (filtros de alta eficiencia) y dispositivos de desinfección que integren cámaras germicidas con luz ultravioleta de onda corta UVc (200-280 nm) las cuales son sugeridas por Fredes (2020) y la Asociación Internacional Ultravioleta (IUVA) como potente desinfectante que inactiva patógenos como virus y bacterias.

\section{Conclusiones}

Se puede concluir que se deben tomar acciones correctivas en hospitales, clínicas, EPS e instituciones de salud verificando, diseñando o rediseñando sus sistemas de acondicionamiento de aire, calefacción y ventilación bajos los estándares 170-2013 de ASHRAE/ASHE, ASHRAE 55 y 62, DIN 1946 e ISO 29463. Asi mismo deben evaluar la posibilidad de reemplazar sus sistemas de filtración de aire por dispositivos o equipos que incorporen lámparas ultravioletas (UVc), sistemas de pre-filtros y filtros HEPA que permitan retener y ofrecer efecto germicida que garanticen aire higienizado y sin SARS-CoV-2 presente en sus partículas o moléculas.

\section{Referencias}

Ahumada Villafañe, I., Escudero Sabogal, I., \& Gutiérrez Jaraba, J. (2016). NORMATIVIDAD DE RIESGOS LABORALES EN COLOMBIA Y SU IMPACTO EN EL SECTOR DE HIDROCARBUROS. IPSA SCIENTIA: Revista Científica Multidisciplinaria, 1(1), 31-42.

IPSA SCIENTIA - Vol. 5 № $1-2020$ 
Ahumada Villafañe, I., Palacio Angulo, J., Posada Lopez, J., \& Orjuela, I. D. (2019). PERCEPCIÓN DEL RIESGO LABORAL EN TRABAJADORES OPERATIVOS DEL SECTOR METALMECÁNICO. IPSA SCIENTIA: Revista Científica Multidisciplinaria, 4(1), 49-59.

Andersen, K. G., Rambaut, A., Lipkin, W. I., Holmes, E. C., \& Garry, R. F. (2020). The proximal origin of SARS-CoV2. Nature medicine, 26(4), 450-452.

ASHE: American society for health care engineering (https://www.ashe.org/).

ASHRAE Standard. (2017). ASHRAE Standard 52.2-2017, Method of testing general ventilation air-cleaning devices for removal efficiency by particle size. Atlanta: American Society of Heating, Refrigerating and Air-Conditioning Engineers.

ASHRAE, A. (2013). ANSI/ASHRAE/ASHE Standard 170-2013. Ventilation of Health Care Facilities. American Society of Heating, Refrigerating and Air-Conditioning Engineers, Inc, Atlanta.

ASHRAE: American Society of Heating, Refrigerating and Air-Conditioning Engineers (https://www.ashrae.org/).

Barrios Monges, Laurentino. (2020). Consideraciones acerca de la pandemia de covid-19. Anales de la Facultad de Ciencias Médicas (Asunción), 53(1), 13-16.

Beam, Elizabeth L. et al. Personal protective equipment processes and rationale for the Nebraska Biocontainment Unit during the 2014 activations for Ebola virus disease American Journal of Infection Control, Volume 44, Issue 3, 340 - 342.

Bourouiba L. Turbulent Gas Clouds and Respiratory Pathogen Emissions: Potential Implications for Reducing Transmission of COVID-19. JAMA 2020 Mar 26[Online ahead of print].

Cortés, Manuel E.. (2020). Coronavirus como amenaza a la salud pública. Revista médica de Chile, 148(1), 124-126.

De Dear, R. J., \& Brager, G. S. (2002). Thermal comfort in naturally ventilated buildings: revisions to ASHRAE Standard 55. Energy and buildings, 34(6), 549-561.

Dong, L., Tian, J., He, S., Zhu, C., Wang, J., Liu, C., \& Yang, J. (2020). Possible vertical transmission of SARS-CoV-2 from an infected mother to her newborn. Jama, 323(18), 1846-1848.

Franco-Paredes, Carlos, Kuri-Morales, Pablo, Alvarez-Lucas, Carlos, Palacios-Zavala, Ethel, Nava-Frías, Margarita, Betancourt-Cravioto, Miguel, Santos-Preciado, José Ignacio, \& Tapia-Conyer, Roberto. (2003). Síndrome agudo respiratorio severo: un panorama mundial de la epidemia. Salud Pública de México, 45(3), 211-220.

Fredes, Pablo. (2020). Recomendaciones para la selección y operación de dispositivos de desinfección UV para aire y superficies. Preparado por la Asociación Internacional Ultravioleta (IUVA). 10.13140/RG.2.2.21622.75849.

García, A. T. (2020). Un mundo, una salud: la epidemia por el nuevo coronavirus COVID-19. Medicina clínica, 154(5), 175177.

Herrán Villalba, V., Lugo Calderón, E., Puello Martínez, A., \& Ruiz Restrepo, A. (2016). NIVEL DE CONOCIMIENTO EN RIESGOS LABORALES: EL CASO DE TRABAJADORES DE SERVICIOS GENERALES EN UNA UNIVERSIDAD. IPSA SCIENTIA: Revista Científica Multidisciplinaria, l(1), 43-51.

IPSA SCIENTIA - Vol. 5 № $1-2020$ 
Huang, C., Wang, Y., Li, X., Ren, L., Zhao, J., Hu, Y., ... \& Cheng, Z. (2020). Clinical features of patients infected with 2019 novel coronavirus in Wuhan, China. The lancet, 395(10223), 497-506.

Jaimes Morales, J. (2018). SISTEMA DE GESTIÓN DE SEGURIDAD Y SALUD EN EL TRABAJO: UNA REVISIÓN DESDE LOS PLANES DE EMERGENCIA. IPSA SCIENTIA: Revista Cientifica Multidisciplinaria, 3(1), 23-29.

Johnson GR, Morawska L, Ristovski, ZD, et al. Modality of human expired aerosol size 10 distributions. Journal of Aerosol Science. 2011; 42: 839-851. doi:10.1016/j.jaerosci.2011.07.009.

Kissler, S. M., Tedijanto, C., Goldstein, E., Grad, Y. H., \& Lipsitch, M. (2020). Projecting the transmission dynamics of SARSCoV-2 through the postpandemic period. Science, 368(6493), 860-868.

Kissler, S. M., Tedijanto, C., Goldstein, E., Grad, Y. H., \& Lipsitch, M. (2020). Projecting the transmission dynamics of SARSCoV-2 through the postpandemic period. Science, 368(6493), 860-868.

Lee J, Yoo D, Ryu S et al., Quantity, size distribution, and characteristics of cough generated aerosol produced by patients with an upper respiratory tract infection. Aerosol and Air Quality Research. 2019; 19: 840-853.

Li Y, Qian H, Hang J, Chen X, Hong L, Liang P, et al. Evidence for probable aerosol transmission of SARS-CoV-2 in a poorly ventilated restaurant. medRxiv [Internet]. 22 de abril de 2020 [citado 6 de mayo de 2020];2020.04.16.20067728. Disponible en: https://www.medrxiv.org/content/10.1101/2020.04.16.20067728v1.

Lu, X., Zhang, L., Du, H., Zhang, J., Li, Y. Y., Qu, J., ... \& Wu, C. (2020). SARS-CoV-2 infection in children. New England Journal of Medicine, 382(17), 1663-1665.

Ludert, Juan Ernesto, \& Franco Cortés, Manuel Antonio. (2020). La pandemia de COVID-19, ¿qué podemos aprender para la próxima?. Universitas Medica, 61(3), 1-3.

Marrugo Ligardo, Y. (2017). HEALTH AND SAFETY MANAGEMENT SYSTEM IN LATIN AMERICA: A REVIEW FROM THE HSEQ INTEGRATION. IPSA SCIENTIA: Revista Científica Multidisciplinaria, 2(1), 38-45.

Moreno-Montoya, José. (2020). El desafío de comunicar y controlar la epidemia por coronavirus. Biomédica, 40(1), 11-13.

Moschandreas, D. J., \& Vuilleumier, K. L. (1999). ETS levels in hospitality environments satisfying ASHRAE standard 621989:"ventilation for acceptable indoor air quality". Atmospheric Environment, 33(26), 4327-4340.

Olesen, B. W., \& Brager, G. S. (2004). A better way to predict comfort: The new ASHRAE standard 55-2004.

OMS (World Health Organization). (2020). Modos de transmisión del virus que causa la COVID-19. Disponible en: https://www.who.int/es/news-room/commentaries/detail/modes-of-transmission-of-virus-causing-covid-19-implications-foripc-precaution-recommendations.

Palacio Angulo, J., Ahumada Villafañe, I., Orjuela, I. D., \& Posada Lopez, J. (2018). TOWARDS A STRATEGIC EPIDEMIOLOGY OF OCCUPATIONAL SAFETY AND HEALTH. IPSA SCIENTIA: Revista Científica Multidisciplinaria, 3(1), 30-37.

Palacios Cruz, M., Santos, E., Velázquez Cervantes, M. A., \& León Juárez, M. (2020). COVID-19, a worldwide public health emergency. COVID-19, una emergencia de salud pública mundial. Revista clínica española, S0014-2565(20)30092-8. Advance online publication. https://doi.org/10.1016/j.rce.2020.03.001.

IPSA SCIENTIA - Vol. 5 № 1 - 2020 
Pan, Y., Zhang, D., Yang, P., Poon, L. L., \& Wang, Q. (2020). Viral load of SARS-CoV-2 in clinical samples. The Lancet Infectious Diseases, 20(4), 411-412.

Paules, C. I., Marston, H. D., \& Fauci, A. S. (2020). Coronavirus infections-more than just the common cold. Jama, 323(8), 707-708.

Persily, A. (2015). Challenges in developing ventilation and indoor air quality standards: The story of ASHRAE Standard 62. Building and Environment, 100(91), 61-69.

Quintana Salcedo, A., Ramos De La Espriella, C., \& Lugo Calderón , E. (2019). RIESGO BIOLÓGICO GENERADO POR EL MANEJO DE RESIDUOS SÓLIDOS EN UN CENTRO HOSPITALARIO. IPSA SCIENTIA: Revista Científica Multidisciplinaria, 4(1), 33-48.

Santarpia J. Transmission Potential of SARS-CoV-2 in Viral Shedding Observed at the University of Nebraska Medical Center preprints from medRxiv and bioRxiv (2020). doi: $\underline{10.1101 / 2020.03 .23 .20039446}$.

Serra Valdés, Miguel Ángel. (2020). Infección respiratoria aguda por COVID-19: una amenaza evidente. Revista Habanera de Ciencias Médicas, 19(1), 1-5.

Sia, S. F., Yan, L. M., Chin, A. W., Fung, K., Choy, K. T., Wong, A. Y., ... \& Peiris, M. (2020). Pathogenesis and transmission of SARS-CoV-2 in golden hamsters. Nature, 583(7818), 834-838.

Tellier, R., Li, Y., Cowling, B.J. et al. Recognition of aerosol transmission of infectious agents: a commentary. BMC Infect Dis 19, 101 (2019). https://doi.org/10.1186/s12879-019-3707-y

Tong, Z. D., Tang, A., Li, K. F., Li, P., Wang, H. L., Yi, J. P., ... \& Yan, J. B. (2020). Potential presymptomatic transmission of SARS-CoV-2, Zhejiang province, China, 2020. Emerging infectious diseases, 26(5), 1052.

Trilla, A. (2020). Un mundo, una salud: la epidemia por el nuevo coronavirus COVID-19. Med Clin (Barc), 154(5), 175-177.

Van Doremalen N, Bushmaker T, Morris D, et al. Aerosol and surface stability of HCoV-19 (SARS-CoV-2) compared to SARS-CoV-1. New England Journal of Medicine. Published online March 17, 2020. DOI: 10.1056/NEJMc2004973.

Van Doremalen, N., Bushmaker, T., Morris, D. H., Holbrook, M. G., Gamble, A., Williamson, B. N., ... \& Lloyd-Smith, J. O. (2020). Aerosol and surface stability of SARS-CoV-2 as compared with SARS-CoV-1. New England Journal of Medicine, 382(16), 1564-1567.

Wang, W., Xu, Y., Gao, R., Lu, R., Han, K., Wu, G., \& Tan, W. (2020). Detection of SARS-CoV-2 in different types of clinical specimens. Jama, 323(18), 1843-1844.

Yeo, C., Kaushal, S., \& Yeo, D. (2020). Enteric involvement of coronaviruses: is faecal-oral transmission of SARS-CoV-2 possible?. The lancet Gastroenterology \& hepatology, 5(4), 335-337.

Zhou, B., \& Shen, J. (2007). Comparison of general ventilation air filter test standards between America and Europe. Proceedings of IAQVEC, 639-646.

Zou, L., Ruan, F., Huang, M., Liang, L., Huang, H., Hong, Z., ... \& Guo, Q. (2020). SARS-CoV-2 viral load in upper respiratory specimens of infected patients. New England Journal of Medicine, 382(12), 1177-1179.

IPSA SCIENTIA - Vol. 5 № 1 - 2020 